\title{
Multimicrobial Pneumonia during the Early Stages of a Global Pandemic
}

\section{J alal $\mathbf{H}^{1 *}$ and Henriksen $\mathbf{G}^{2}$}

${ }^{1}$ Department of Medical Education/ Family Medicine, Good Samaritan Hospital Medical Center, USA ${ }^{2}$ Department of Family Medicine, Good Samaritan Hospital Medical Center, USA

*Corresponding author: Hamza J alal, Department of Medical Education/ Family Medicine, Good Samaritan Hospital Medical Center, USA

Received: J une 10, 2021; Accepted: August 03, 2021; Published: August 10, 2021

\begin{abstract}
Community-acquired pneumonia is an acute infection of lung parenchyma which causes local and systemic inflammatory changes via cytokines. Several bacteria and viruses are responsible for this type of pneumonia, and the most common bacterial cause is Streptococcus pneumoniae. The classic symptoms are cough, fever, and pleuritic chest pain. In the Winter of 2020, a new strain of coronavirus known as SARS-CoV-2 spread throughout the world and was responsible for a global pandemic that transformed the way we live our lives.

A 93-year old female presented to the hospital with respiratory distress and was found to have not only COVID-19 pneumonia but also superimposed Methicillin-resistant Staphylococcus aureus and Pseudomonas aeruginosa pneumonia. Following the most up-to-date guidelines, she was determined to have community-acquired pneumonia. Methicillin-resistant Staphylococcus aureus and Pseudomonas aeruginosa are uncommon causes of communityacquired pneumonia. She was treated with the standard of care at the time, which included vancomycin, piperacillin-tazobactam, and hydroxychloroquine.

This case highlights the rarity of this specific presentation of community acquired pneumonia in regards to microbial etiology. It showcases that patients may develop certain diseases despite not having any risk factors. A major takeaway point is that apt decision making is a critical and time sensitive matter when determining whether a bacterial co-infection is present since it can affect patient outcomes. Since co-infections are relatively infrequent, antibiotic use in COVID-19 positive patients needs to be tailored accordingly. At the same time, it is crucial to keep in mind that co-infections are associated with increased severity of COVID-19 as well as poorer outcomes.
\end{abstract}

Keywords: COVID-19; Pseudomonas aeruginosa; MRSA; Communityacquired pneumonia; Pandemic

\section{Case Presentation}

In March of 2020, a 93-year-old female with a medical history significant for atrial fibrillation, hypertension, hyperlipidemia, mild dementia, and Percutaneous Endoscopic Gastrostomy (PEG) tube placement presented to the emergency room from a nursing facility with respiratory distress and productive cough.

Vital signs included a temperature of 102.5 degrees Fahrenheit, blood pressure of $96 / 60 \mathrm{mmHg}$, heart rate of 130 beats per minute, respiratory rate of 36 breaths per minute, body mass index of 26.57 $\mathrm{kg}$ per $\mathrm{m} 2$, and oxygen saturation of $99 \%$. Blood pressure deteriorated rapidly during her initial assessment in the emergency room to $82 / 56$ mmHg.

Physical examination revealed an ill-appearing elderly female in respiratory distress with an increased respiratory rate and effort, use of accessory muscles, rhonchorous breath sounds, and rales throughout bilateral lung fields. The abdomen was soft, non distended with PEG tube placement in the anterior abdominal wall. She was lethargic and unable to answer questions. Portable bedside chest $\mathrm{x}$-ray demonstrated bilateral interstitial infiltrates.

Due to respiratory distress and tachypnea, the patient was intubated. The patient was started on intravenous fluids as she was hypotensive and met sepsis criteria. Despite adequate weightbased intravenous fluids, she remained hypotensive and was started on norepinephrine due to septic shock. Due to concern for concurrent bacterial and COVID-19 infection, the patient was started on IV vancomycin, IV piperacillin and tazobactam, and hydroxychloroquine.

The initial laboratory results revealed a white blood cell count of 10,210, hemoglobin of $14.1 \mathrm{~g}$ per dl, platelet count of 178,000 . The patient also had lymphopenia. Sodium was $134 \mathrm{mmol}$ per $\mathrm{L}$, potassium was $4.5 \mathrm{mmol}$ per $\mathrm{L}$, chloride was $99 \mathrm{mmol}$ per $\mathrm{L}$, bicarbonate was $26 \mathrm{mmol}$ per L, BUN was $25 \mathrm{mg}$ per $\mathrm{dl}$, creatinine was $0.9 \mathrm{mg}$ per $\mathrm{dl}$, glucose was $143 \mathrm{mg}$ per $\mathrm{dl}$, albumin was $2.8 \mathrm{~g}$ per $\mathrm{dl}$, aspartate transaminase was 56 unit per $\mathrm{L}$ and alanine transaminase was 61 unit per $\mathrm{L}$, lactic acid was $5.7 \mathrm{mmol}$ per L, and procalcitonin was $4.61 \mathrm{ng}$ per $\mathrm{mL}$. Inflammatory markers were also elevated and included ferritin (286 ng per L), C reactive protein ( $7.6 \mathrm{mg}$ per L), and lactate dehydrogenase (308 unit per L).

There was high clinical suspicion for COVID-19, and as expected, the patient tested positive for SARS Coronavirus CoV-2 via nasal PCR. What was unexpected was that the patient also tested positive for 
Pseudomonas aeruginosa and Methicillin-Resistant Staphylococcus Aureus (MRSA) via respiratory culture.

On day 12 of her hospital admission, she was extubated and placed on a $100 \%$ nonrebreather mask. She was not tolerating extubation as her oxygen demands were increasing. After a discussion with the primary team, the intensivist and palliative care team, the family decided against re-intubation. On day 14 of admission, the patient expired.

\section{Discussion/Conclusion}

COVID-19 pneumonia has become a significant cause of Community-Acquired Pneumonia (CAP) in the past year. However, Pseudomonas aeruginosa and MRSA are not common causes of CAP, and this component made this patient's presentation unique [1]. There were no other reported or published cases of this "triple pneumonia." A retrospective cohort study examined 585 ward patients and 145 ICU patients with CAP. Of the 585 ward patients, 12 patients (2.1\%) had Pseudomonas, and seven patients (1.2\%) had MRSA. Regarding the ICU patients, eight patients (5.5\%) had Pseudomonas, and three patients (2.1\%) had MRSA [2]. Another study evaluated 2259 patients with CAP requiring hospitalization in Chicago and Nashville. Eight patients $(0.3 \%)$ tested positive for Pseudomonas, 37 patients (1.6\%) tested positive for MRSA, and only 115 patients (5.1\%) were positive for polymicrobial infections [3].

Further highlighting the infrequent occurrence of bacterial coinfections, a review article that analyzed 28 observational studies and 21 case reports found that in hospitalized COVID-19 patients, the incidence of secondary pulmonary bacterial infections was low at $16 \%[4]$.

Our patient did not have the common risk factors associated with these two bacterial pathogens. Influenza, end-stage renal disease, Chronic Obstructive Pulmonary Disease (COPD), lung abscess, and illicit drug use are risk factors for MRSA. Bronchiectasis, COPD, and lung abscess are risk factors for Pseudomonas [5]. Our patient came from a nursing home; however "health care-associated pneumonia" is an outdated term, and that nomenclature is no longer used. Therefore she was diagnosed with community-acquired pneumonia. Nasogastric tube rather than PEG tube predisposes a patient to aspiration pneumonia [6]. She was not hospitalized in the threemonth span prior to her admission, nor did she receive intravenous antibiotics in that same time frame, which are other risk factors for MRSA and Pseudomonas [7].

COVID-19 and bacterial co-infections are not common. A team of researchers evaluated 30 studies which included 3834 patients, and only $7 \%$ of hospitalized COVID-19 patients had a bacterial co-infection, and the most common organisms were Mycoplasma pneumonia, Pseudomonas aeruginosa, and Haemophilus influenzae [8].

MRSA and Pseudomonas aeruginosa pneumonia on their own are associated with worse outcomes and higher rates of mortality. There is also an increased risk of sepsis, ICU admission, and mechanical ventilation, all of which the patient had. The inoculation of three virulent pathogens certainly led to the poor outcome of this patient. Identifying co-infections is important because COVID-19 patients with a coinfection were more likely to die than patients who did not have a coinfection [9].

A research article assessed 905 COVID-19 positive patients and approximately $9.5 \%$ had confirmed bacterial co-infections. Patients with advanced age, cardiovascular comorbidities, increased body temperature, increased neutrophils count, decreased lymphocytes, elevated procalcitonin, and decreased albumin were at increased risk for bacterial co-infections [10].

This patient was affected by COVID-19 during the infancy of the global pandemic where there was a lack of valid, evidencebased medicine, and there were no clear guidelines set in place. The majority of the treatment was supportive and anecdotal. IV vancomycin and IV piperacillin-tazobactam were appropriate for the coverage of MRSA and Pseudomonas aeruginosa pneumonia, respectively. However, the use of hydroxychloroquine would later be determined to have insufficient evidence. This patient did not receive corticosteroids because, at that point in time, the recommendations were to avoid corticosteroids. Now we know that corticosteroids are beneficial and recommended in COVID-19 positive patients requiring supplemental oxygenation.

The rarity of this "triple pneumonia" made this case distinct and unparalleled from the other COVID cases that were rampant during the global pandemic. Despite not having any risk factors for MRSA or Pseudomonas, the patient was positive for both. A physician must be prudent when assessing COVID-19 positive patients for bacterial co-infections. Although these co-infections are infrequent, they can negatively impact patient outcome, survival and recovery. The presence of multiple microbial pathogens in a COVID-19 patient can be detrimental therefore it is important to evaluate patients for coinfections in order to properly treat the patient.

\section{References}

1. Johansson N, Kalin M, Tiveljung-Lindell A, Giske CG, Hedlund J. Etiology of community-acquired pneumonia: increased microbiological yield with new diagnostic methods. Clin Infect Dis. 2010; 50: 202-209.

2. Restrepo MI, Mortensen EM, Velez JA, Frei C, Anzueto A. A comparative study of community-acquired pneumonia patients admitted to the ward and the ICU. Chest. 2008; 133: 610-617.

3. Jain S, Self WH, Wunderink RG, Fakhran S, Balk R, Bramley AM, et al. CDC EPIC Study Team. Community-Acquired Pneumonia Requiring Hospitalization among U.S. Adults. N Engl J Med. 2015; 373: 415-427.

4. Chong WH, Saha BK, Ramani A, et al. State-of-the-art review of secondary pulmonary infections in patients with COVID-19 pneumonia. Infection. 2021.

5. Lewis PO. Risk Factor Evaluation for Methicillin-Resistant Staphylococcus aureus and Pseudomonas aeruginosa in Community-Acquired Pneumonia. Ann Pharmacother. 2021; 55: 36-43.

6. Marik PE. Aspiration pneumonitis and aspiration pneumonia. N Engl J Med. 2001; 344: 665-671.

7. Kalil AC, Metersky ML, Klompas M, et al. Management of Adults With Hospital-acquired and Ventilator-associated Pneumonia: 2016 Clinical Practice Guidelines by the Infectious Diseases Society of America and the American Thoracic Society. Clin Infect Dis. 2016; 63: e61.

8. Lansbury L, Lim B, Baskaran V, Lim WS. Co-infections in people with COVID-19: a systematic review and meta-analysis. J Infect. 2020; 81: 266275.

9. Lansbury, Louise, et al. "Co-Infections in People with COVID-19: a Systematic Review and Meta-Analysis." Journal of Infection. 2020; 81: 266-275.

10. He S, Liu W, Jiang M, Huang P, Xiang Z, Deng D, et al. Clinical characteristics of COVID-19 patients with clinically diagnosed bacterial co-infection: A multicenter study. PLoS One. 2021; 16: e0249668. 